\title{
Mobile and wireless technologies applying on sphygmomanometer and pulsimeter for patients with pacemaker implementation and other cardiovascular complications
}

\author{
Ching-Sung Wang
}

Department of Electronic Engineering Oriental Institute of Technology, Taipei, Taiwan, China. Emill: ff020@mail.oit.edu.tw

Received 11 August 2009; revised 9 September 2009; accepted 10 September 2009.

\begin{abstract}
Continuously monitoring pulse is very important for the pacemaker patients., and the continuously observing blood pressure is also a matter of concern for those who have hypertension, coronary heart disease, or other cardiovascular diseases, for example cardiac arrhythmia and hypertension. What we expect is not only to eliminate arrhythmia, also to treat patients as a whole of body. Therefore, how to keeping monitoring blood pressure and pulses rise to a very important issue. This research edits a wrist-able sphygmomanometer and pulsimeter combining with cell phone, to achieve wireless, continuous, and real-time observation, early detects any accident occurring from the patients with pacemaker implementation or with other cardiovascular complications
\end{abstract}

Keywords: Pacemaker; Hypertension; Coronary Heart Disease; Cardiovascular Disease; Sphygmomanometer and Pulsimeter

\section{INTRODUCTION}

The population for pacemaker implant is not limited by age, sex, or race. Over 100,000 pacemakers are implanted per year in the United States. Approximately 500,000 Americans have an implantable permanent pacemaker device [1]. Pacemaker can be affected by electromagnetic interference in several different ways, including temporary inhibition of the pacemaker, temporary function at the fixed noise rate, temporary function at the fixed magnet rate, permanent inhibition or malfunction, and random reprogramming. For any of these results to occur, the $\mathrm{E}$ field strength must be greater than $200 \mathrm{~V} / \mathrm{m}$ or the magnetic field strength must be greater than 10 Gauss [2].

Cellular telephones can interfere with the function of implanted cardiac pacemakers $[3,4,5]$. However, when telephones are placed over the ear, the normal position, this interference does not pose a health risk [5]. Barbaro $\mathrm{V}$, et al. The research of influence between GSM mobile phone and implanted pacemaker indicates that electromagnetic interference effects were detected at a maximum distance of $10 \mathrm{~cm}$ with the pacemaker programmed at its minimum sensing threshold. When the phone antenna was in direct contact with patient's skin over the implant, electromagnetic interference effects occurred at maximum ventricular and a trial sensing thresholds of 4 $\mathrm{mV}$ and $2.5 \mathrm{mV}$, respectively [6]. Therefore, decreasing wireless emission power and rising pacemaker emission distance are the most useful methods of lowering the interference of magnetic to the pacemaker.

In order not to affect users' habits of using mobile and to avoid the electromagnetic interference effects of pacemaker, we design a wrist-able sphygmomanometer and pulsimeter which is using the Bluetooth power class II $[7,8]$ to be the device for short-distance transmission. Furthermore, to combine with a cellular phone, we can do the prolonged distance observation and treatment immediately for the patients with setting pacemaker or with other cardiovascular disease.

\section{SYSTEM ARCHITECTURE}

This research aims at designing wrist-able sphygmomanometer and pulsimeter. We design a non-invasive sphygmomanometer [9] to remind the user to measure the blood pressure every hour. Pulsimeter is an electronic device which is based on pressure sensor, and can observe pulse continuously. In order to avoid the influence of electromagnetic wave on the pacemaker, this research is using the Bluetooth power class II equipment to shorten the distance which wireless data transmission device of sphygmomanometer transmits to client-end cellular phone, then use the GPRS (General Packet Radio Service) $[10,11]$ to do the long distance data trans- 


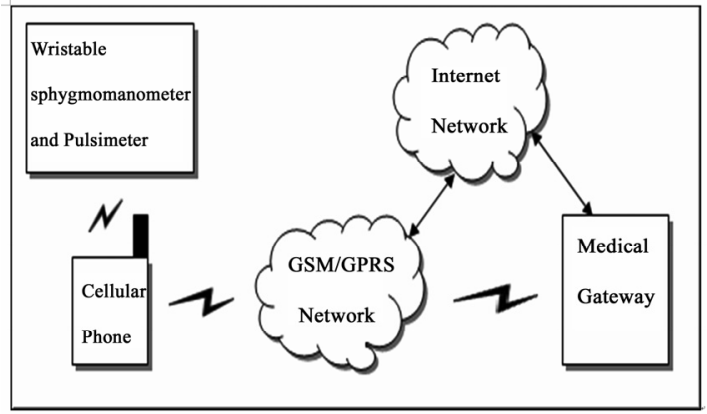

Figure 1. System architecture.

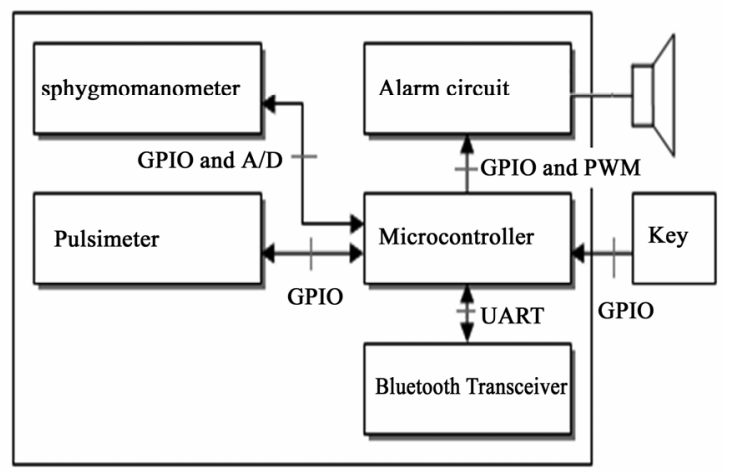

Figure 2. Wristable sphygmomanometer and pulsimeter hardware architecture.

mission. This can transmit blood pressure and pulse rate to Remote Medical Server and then provide them to specialist doctors for reference data.

Because of the connecting method of GPRS is a packet switch [10,11], it doesn't connect with Remote Medical Server continuously. Therefore, this research designs that Remote Medical Server used GSM (Global System for Mobile Communications) circuit connection(voice connection) $[10,11,12]$ to inform wrist-able sphygmomanometer and pulsimeter, and drives sphygmomanometer to measure blood pressure immediately. Then, the sphygmomanometer will send the outcome of blood pressure to Remote Medical Server by GPRS $[10,11,13]$. Figure 1 details the block diagram of system architecture.

\section{HARDWARE ARCHITECTURE}

The hardware system of this research is divided into two parts, one is wrist-able sphygmomanometer and pulsimeter; the other one is client-end cellular phone. The below is the detail of them.

\subsection{Wristable Sphygmomanometer and Pulsimeter}

Figure 2 details the block diagram of wrist-able sphygmomanometer and pulsimeter hardware architecture. The Controller unit of wrist-able sphygmomanometer

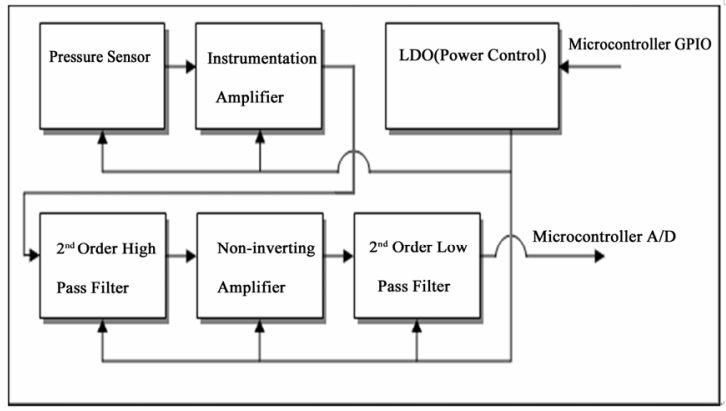

Figure 3. Sphygmomanometer hardware architecture.

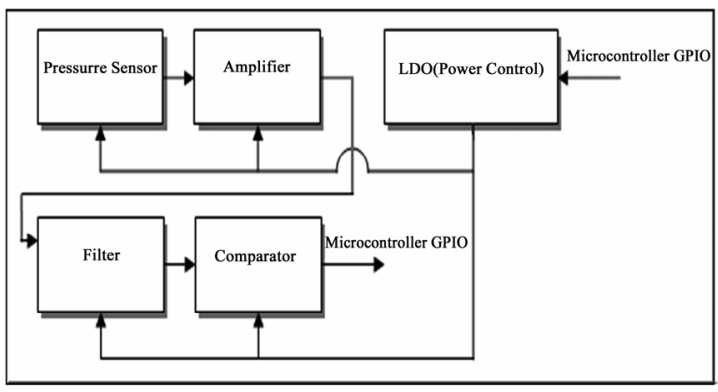

Figure 4. Pulsimeter hardware architecture.

and pulsimeter is microcontroller [14], which controls and reads the peripheral units. Here is the description of each unit.

1) Sphygmomanometer $[9,14,15,16]$ : The sphygmomanometer includes LDO (Low Dropout Regulators) as power controller, pressure sensor, filter, amplifier circuit and accessories. The research is using the pressure sensor of SCC series which is manufactured by HoneyWell company. The pressure sensor will transmit different message according to the changing pressure. The message passes through the procedures of magnifying (magnify the micro-message form sensor), and filtering (remove noise), then all the analog messages will be sent to the microcontroller via A/D (Analog/Digital) interface. Figure 3 shows the block diagram of sphygmomanometer hardware architecture.

2) Pulsimeter $[9,14,15,16]:$ The pulsimeter includes LDO, pressure sensor, filter, amplifier circuit and accessories. The circuit is using the SCC series of HonyWell company, but it is a different sensitivity pressure sensor. Putting sensor pad on pulse of hand, the pressure sensor will convert the normal pulse beat to a larger voltage output by magnifying, filtering, comparing (transform to digital signal), and then inputs it into microcontroller. Figure 4 is shown the block diagram of pulsimeter.

3) Bluetooth transceiver [14,17]: The project uses $\mathrm{BC} 3$ made by CSR as the Bluetooth transceiver. The microcontroller communicates with the Bluetooth by UART (Universal asynchronous receiver/transmitter) interface and controls all the process by software.

4) Alarm circuit: The main purpose of this part is to 


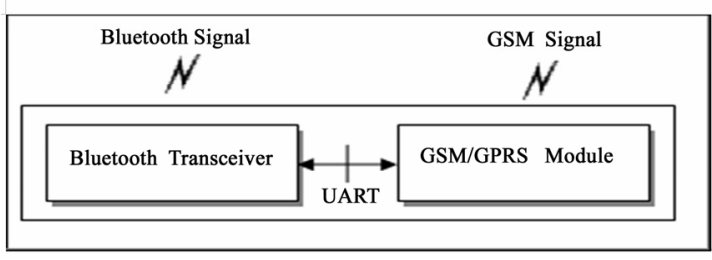

Figure 5. Client-end Cellular Phone Hardware Architecture.

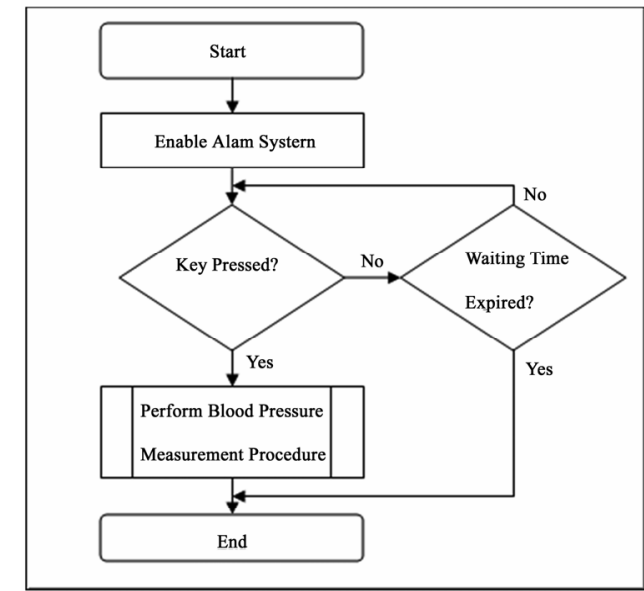

Figure 6. The software algorithm of continuous pulse and blood measuring and monitoring procedure.

send a notice to the users, and then to perform blood pressure measurement. Microcontroller can activate the alarm circuit every hour. However, on the other one end, Remote Medical Server can prescribe the blood pressure measurement, so that the users can measure their blood pressure when they require.

\subsection{Client-End Cellular Phone Hardware Architecture}

Figure 5 details the block diagram of client-end cellular phone hardware architecture, which includes two main parts, Bluetooth and GSM/GPRS.

1) Bluetooth transceiver $[14,17,18]$ : The hardware framework is similar to the Bluetooth transceiver of wrist-able sphygmomanometer and pulsimeter. It communicates with GSM/GPRS module by UART interface.

2) GSM Module [18]: It includes Baseband part and RF part. The main components are Baseband IC (including DSP), TFT-LCD, NOR Flash, SRAM, Power Management IC and RF IC. We use MT6219 made by MediaTek as the platform of the GSM/GPRS system.

\section{SOFTWARE ALGORITHM}

\subsection{Continuous Pulse and Blood Measuring and Monitoring Procedure}

Pulse monitor procedure $[14,15,16,19]$ : Microcontroller

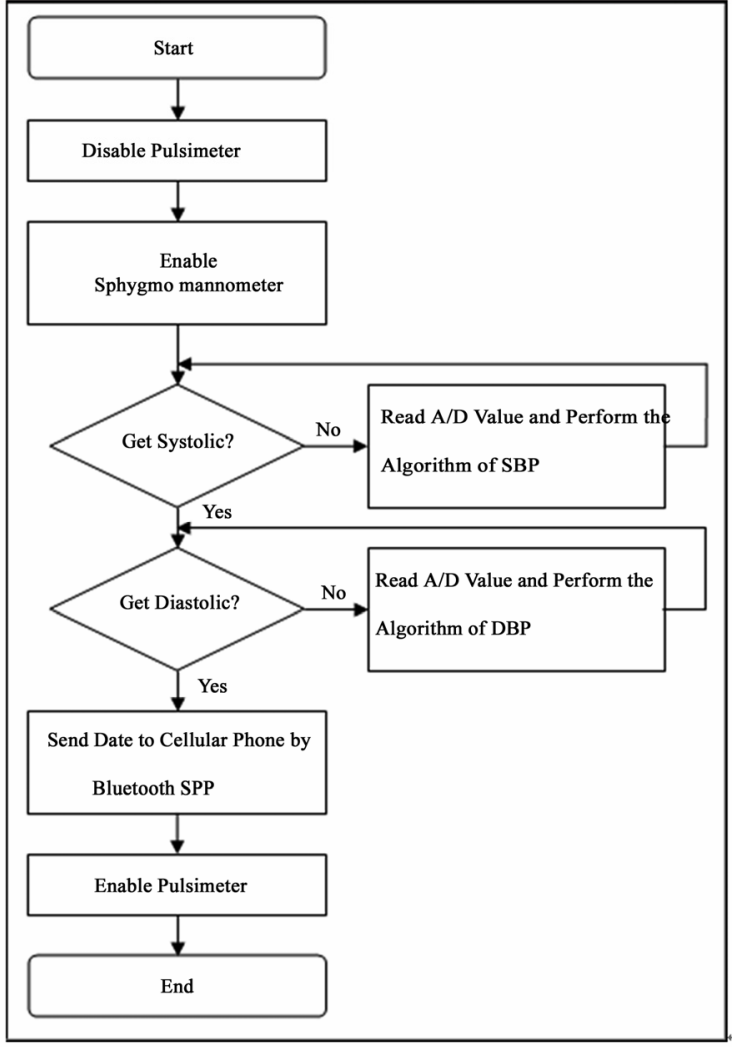

Figure 7. The software algorithm of measure blood presure procedure.

reads the pulsimeter every minute, then transmits the results to the client-end cellular phone by Bluetooth SPP (Serial Port Profile).

Blood pressure measure procedures [9,14,15,16,19]: Microcontroller enables alarm system to inform user every hour that the time requires to measure the blood pressure. Users need to put hand on the proper position so that sphygmomanometer can have a better detection. While the users put hand on proper position and push trigger button, then the microcontroller controls pulsimeter's LDO to stop action and start to measure the blood pressure. After that, microcontroller transmits blood pressure to the cellular phone by Bluetooth SPP, and to activate pulsimeter. If the user doesn't do the action of blood pressure measurement, the microcontroller would re-activate pulsimeter after a while. The software algorithm of continuous pulse and blood measurement and the monitoring procedure is shown in Figure 6 and Figure 7.

\subsection{Client-End Clelular Phone Data Handling Procedure}

While the Bluetooth of mobile receives the data from the Bluetooth of wrist-able sphygmomanometer and pulsimeter, it analyzes the data of blood pressure or pulse and to store (includes the measurement time) individu- 


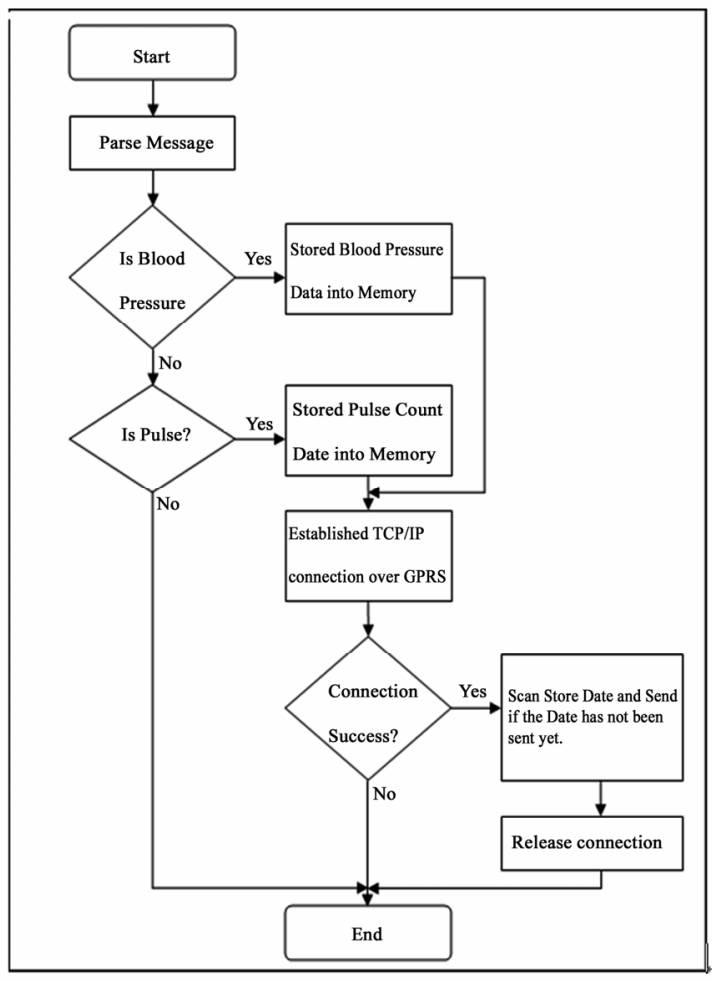

Figure 8. The software algorithm of client-end cellular phone data handling procedure.

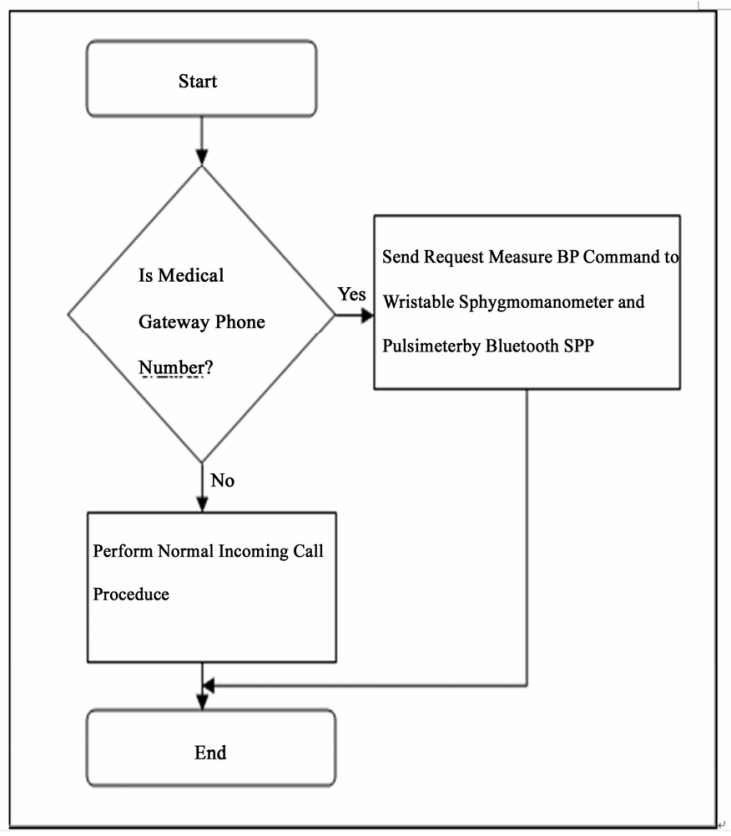

Figure 9. The software algorithm of remote medical server claim for mearuring blood pressure.

ally at first. Finally, it establishes GPRS connection. After successful connecting, mobile scans stored blood pressure and pulse data, and then it transmits to the Remote Medical Server if the data has not been sent yet.
The software algorithm of mobile data handling procedure is shown on Figure 8 [13,19,20,21].

\subsection{Remote Medical Server Claim for Measur- ing Blood Pressure}

If the doctor considers that it is necessary to get the patient's current blood pressure-immediately, he/she can call to client-end cellular phone by GSM module on the Remote Medical Server. While client-end cellular phone receives incoming call form server-end, the Bluetooth SPP transmits command of measuring blood pressure to wrist-able sphygmomanometer and pulsimeter. While wrist-able sphygmomanometer and pulsimeter receive the command from the Remote Medical Server, they activate blood pressure measurement and transmit procedures (Figure 6, 7, 8). The software algorithm of Remote Medical Gateway claims for measuring blood pressure is shown in Figure 9 [13,19,20,21].

\section{CONCLUSIONS}

It is a fatal risk for the patient with artificial pacemaker implementation while the mobile connection is close to the heart. The artificial pacemaker will consider the electromagnetic wave from mobiles is heart signal, then to do the wrong stimulation on muscle fiber of heart ventricle. However, continuous pulse and blood pressure observation is very essential for the patient of arrhythmia with hypertension coronary arterial heart disease or heart failure. Based on this following the Bluetooth power class II RF test specification, we have designed a portable Bluetooth medium. We expect this system will be benefit for the patients of arrhythmia with hypertension coronary arterial heart disease or heart failure and never cause any inconvenience to their daily life.

\section{REFERENCES}

[1] http://www.surgeryencyclopedia.com/La-Pa/Pacemakers. $\underline{\text { html. }}$

[2] Tacey, S. and Ronald, A. (1992) The effects of electromagnetic fields on cardiac pacerkers. IEEE Transactions on Broadcasting, 38.

[3] Barbaro, V., Bartolini, P., Donato, A., et al. (1995) Do European GSM mobile cellular phones pose a potential risk to pacemaker patients? PACE, 18, 1218-24.

[4] Okan, E., MD, (2002) Electromagnetic interference on pacemakers. Indian Pacing Electrophysiol J., 2(3), 74-78.

[5] Hayes, D.L., Wang, P.J., Reynolds, D.W., et al. (1997) Interference with cardiac pacemakers by cellular telephones. N. Eng. J. Med, 336, 1473-9.

[6] Bluetooth, (2004) Specification of the bluetooth system core. Version 2.0.

[7] Bluetooth, (2004) Radio frequency test suite structure (TSS) and test purposes (TP) specification 1.2, Revision 1.2.3.

[8] Nissila, S., Sorvisto, M., Sorvoja, H., Vieri-Gashi, E. and Myllyla, R. (1998) Non-invasive blood pressure measurement based on the electronic palpation method. 
Engineering in Medicine and Biology Society, Proceedings of the 20th Annual International Conference of the IEEE, 4, 1723-1726.

[9] Lin, Y.B. and Imrich, C. (2000) Wireless and mobile network architectures, Wiley Computer Publishing, 1 Edition (October 2).

[10] Michel, M., Marie-Bernadette, P. (1992) The GSM System for Mobile Communications, Telecom Publishing.

[11] Wang, C.S., Lee, J.H. and Chu Y.T. (2007) Mobile telemedicine application and technologies on GSM, bioinformatics and biomedical engineering, ICBBE, The 1st International Conference on 6-8 July 2007, 1125-1128.

[12] (2004)MediaTek, AT command set, Revision: 0.03 .

[13] (2005) National Semiconductor, SC14440/431/432, SC14435, SC14436/437/438. Baseband processor for PP/FP DECT and WDCT, V1.1, June 16.
[14] (2004) Champion, CM2838, 300mA Low Esr CMOS LDO with Enable, Rev. 1.1.

[15] (2002) HoneyWell, SSC series pressure sensors 0-5 psi through 0-300 psi.

[16] CSR, (2006) BlueCore3-ROM CSP Product Data Book.

[17] MediaTek, (2004) MT6219 GSM/GPRS Baseband Processor Data Sheet, Revision 1.00.

[18] Bluetooth SIG, (2001) Specification of the Bluetooth System Profile, Part K: 5 Serial Port Profile, Version 1.1.

[19] (2003) MediaTek, Customer Device Driver Document, Revision 0.1 .

[20] PixTel, (2004) Writing Applications Using PixTel MMI Platform, Version 1.3. 